\title{
In Silico Pathway Analysis Predicts Metabolites that are Potential Antimicrobial Targets
}

\author{
Malabika Sarker ${ }^{1}$, Sidharth Chopra ${ }^{2}$, Kristien Mortelmans ${ }^{2}$, Krishna Kodukula ${ }^{3}$, Carolyn Talcott ${ }^{1}$ and Amit K. Galande ${ }^{3 *}$ \\ ${ }^{1}$ Computer Science Laboratory, SRI International, Menlo Park, CA 94025 \\ ${ }^{2}$ Center for Infectious Disease and Biodefense Research, SRI International, Menlo Park, CA 94025 \\ ${ }^{3}$ Center for Advanced Drug Research, SRI International, Harrisonburg, Virginia 22802, USA
}

\begin{abstract}
Antibiotic discovery aimed at conventional targets such as proteins and nucleic acids faces challenges from mutations and antibiotic resistance. Small molecule metabolites, however, can be considered resistant to change, as they do not undergo rapid mutations. Developing analogs or scavengers of essential microbial metabolites as antibiotics is a promising strategy that can delay drug resistance. The objective of this work was to identify microbial metabolites that are most suitable targets for antimicrobial discovery. We performed extensive literature mining and systems level pathway analysis to identify bacterial metabolites that fulfill the criteria for drug targets. The BioCyc interactive metabolic pathway maps and Pathway Tools software were used to corroborate our finding. We identified ten metabolites as potential candidates for developing novel antibiotics. These metabolites are Lipid II, meso-diaminopimelate, pantothenate, shikimate, biotin, L-aspartyl-4-phosphate, dTDP-a-L-rhamnose, UDP-Dgalacto-1,4-furanose, des- $\mathrm{N}$-acetyl mycothiol, and Siroheme. The article describes the selection criteria, analysis of metabolic pathways, and the potential role of each of the ten metabolites in therapeutic intervention as broadspectrum antibiotics with emphasis on $M$. tuberculosis. Our study revealed previously unexplored targets along with metabolites that are well established in antibiotic discovery. Identification of established metabolites strengthen our analyses while the newly discovered metabolites could lead to novel antimicrobials.
\end{abstract}

Keywords: Bacteria; Metabolites; BioCyc; Metabolic pathways; Essentiality; Drug target

\section{Introduction}

Many bacterial infections that were once controlled effectively with antibiotics are becoming increasingly resistant to multiple drugs, leading to treatment failure and death [1]. Mutations are among the most common causes of the development of antibiotic resistance. Under the stress of exposure to a given antibiotic, genes encoding errorprone polymerases are up-regulated, leading to the introduction of mutations that confer antibiotic resistance. In conventional antibiotic therapy, the biopolymers that have been used for drug targeting are prone to mutations that lead to antibiotic resistance. Mutations in the quinolone resistance-determining region of gyrA and the mutation of d-Ala-d-Ala to d-Ala-d-Lac in the cell wall peptidoglycan precursor that confers resistance to vancomycin are classical examples [2]. Unlike biopolymers, such as proteins and nucleic acids, which readily respond to evolutionary pressure, small molecule metabolites can be considered immutable. Targeting selective metabolites that are essential for the survival of a bacterium can thus be a much more robust strategy for therapeutic intervention.

Metabolite analogs have long been used as potent and selective inhibitors of microbial growth. These inhibitory analogs are structurally similar to the microbial metabolites and interfere with the functions of the corresponding native metabolites. Recent studies also indicate that "Bacterial-Metabolite-Likeness" can be used as an effective cheminformatic filter in the design and analysis of pharmaceutical libraries for drug discovery $[1,3,4]$.

Antimicrobials used in the treatment of infectious diseases inhibit essential metabolic pathways that exist in the microbial pathogens but not in the hosts. Examples of drugs that act in this way are the sulfonamides, trimethoprim and sulfamethoxazole, which have for three decades had a central role in the treatment of numerous commonly encountered infections [5]. Sulfamethoxazole is a structural analog of para-aminobenzoic acid that inhibits synthesis of the intermediary dihydrofolic acid from its precursors. Trimethoprim is a structural analog of the pteridine portion of dihydrofolic acid that competitively inhibits dihydrofolate reductase, and thus the production of tetrahydrofolic acid from dihydrofolic acid. This sequential blockade of two enzymes in one pathway results in effective antimicrobial action. Host cells do not synthesize their own folic acid, but obtain it as a vitamin. Since hosts do not make folic acid, they are not affected by these drugs, despite their toxicity for bacteria [5]. Isoniazid, the firstline drug used for tuberculosis treatment, is an analog of pyridoxine (Vitamin B6). Isoniazid inhibits mycolic acid synthesis and pyridoxinecatalyzed reactions in mycobacteria [6]. Para-aminosalicylic acid (PAS), a second-line tuberculosis drug, is an antifolate, similar in activity to the sulfonamides [7]. D-cycloserine, a structural analog of D-alanine, acts as a broad-spectrum antimicrobial by inhibiting alanine racemase and D-alanine ligase, which are involved in peptidoglycan synthesis [8]. Roseoflavin, an analog of flavin mononucleotide (FMN) interferes with the FMN function and obtains antimicrobial activity by directly binding to FMN riboswitch aptamers [9].

The success of these metabolite analogs as antibiotics suggests that efforts to discover similar agents could lead to useful new drugs. In this study, we have performed in-depth literature mining and systems level in silico analysis of metabolite pathways of a broad-spectrum of pathogenic bacteria (M.tuberculosis, E. coli, P. aeruginosa, S. aureus,

${ }^{*}$ Corresponding author: Amit K. Galande, Center for Advanced Drug Research, SRI International, Harrisonburg, Virginia 22802, USA, Tel: (540) 438 6621; Fax: (540) 568 5758; E-mail: amit.galande@sri.com

Received April 04, 2011; Accepted April 20, 2011; Published April 25, 2011

Citation: Sarker M, Chopra S, Mortelmans K, Kodukula K, Talcott C, et al. (2011) In Silico Pathway Analysis Predicts Metabolites that are Potential Antimicrobia Targets. J Comput Sci Syst Biol 4: 021-026. doi:10.4172/jcsb.1000071

Copyright: (C) 2011 Sarker M, et al. This is an open-access article distributed unde the terms of the Creative Commons Attribution License,which permits unrestricted use, distribution, and reproduction in any medium, provided the original author and source are credited. 
S. enterica, S. typhirium, K. pneumoniae, K. aerogenes, and B. subtilis) and the human host to identify novel essential metabolites that can be targeted for developing broad-spectrum antibiotics. The research focused on metabolites for which direct experimental evidence supports the conclusion that they are essential for the growth and survival of the relevant bacteria. To identify potential drug targets, we have further screened these metabolites to eliminate any found in the human host. Comparative computational whole genome metabolic pathway analysis was performed using BioCyc Pathway Tools.

\section{Materials and Methods}

\section{Literature mining}

Comprehensive and intelligent manual literature mining was performed to select the appropriate bacterial metabolites based on the following selection criteria:

a. Whether direct experimental evidence shows that the metabolite is essential for growth of bacteria.

b. Whether the metabolite has one or more important biological functions in the bacteria.

c. Whether the metabolite is absent in the human host.

d. Whether the enzymes of the metabolite biosynthesis have suitable synthetic or natural inhibitors that are antimicrobial.

e. Whether the metabolite is physio-chemically suitable for designing the inhibitors.

f. Whether the metabolite analogs have been tested for antimicrobial activity.

g. Whether the metabolite can be a broad-spectrum target.

\section{Comparative analysis using BioCyc pathway tools cellular} overview

To expand on the results of the literature mining, we made use of the computational software capabilities provided by BioCyc [10]. BioCyc Pathway Tools software produces a pathway-based visualization of cellular biochemical networks, called the cellular overview diagram, which supports interrogation and exploration of system-biology analyses of whole organism. The cellular overview includes metabolic, transport, and signaling pathways, and other membrane and periplasmic proteins. BioCyc provides overview diagrams for more than 200 organisms from bacteria to human beings [11]. Pathway Tools automatically generate a cellular overview diagram for an organism from a Pathway/Genome Database (PGDB) describing the genome and biochemical networks of the organism. A PGDB can in turn be automatically generated from the annotated genome sequence of that organism [12]. The cellular overview diagram can be explored for comparative analyses of the complete metabolic networks of two or more organisms. In the display of an overview for one organism, the software can highlight all reactions that are either shared or not shared with other combinations of organisms for which PGDBs are available. For the present work, the entire metabolic network of human from HumanCyc [13] was compared with the networks of pathogens of interest to search for metabolites that are absent from human beings. Moreover, the reactions around the selected metabolites were compared for their presence or absence among the bacteria of choice to determine whether the metabolites are shared and hence can have broadspectrum action. These comparisons are not performed at the sequence level, since the question of whether the organisms share common enzymatic activities is orthogonal to whether the enzymes that catalyze those activities share sequence similarity. Rather, two organisms are considered to share a reaction if the PGDBs for the organisms both specify that the same enzyme catalyzes that reaction.

\section{Pathway analysis using BioCyc pathway genome database}

The study of a metabolite in the context of the relevant biological pathway is important since it provides knowledge about any alternative compensatory pathway that might exist. Nevertheless, the pathway information depicts the genes of the pathways, especially the ones that encode the enzymes that catalyze both the formation and the consumption of the particular metabolite. This information helps to determine whether that metabolite could be considered a suitable target. BioCyc bacterial metabolic pathways were extensively used for studying the relevant pathways and reactions, including the relevant enzymes. BioCyc provides 673 PGDBs, each containing the predicted metabolic network of an organism, including metabolic pathways, enzymes (and the genes encoding them), metabolites (with structural details), and reaction details [14].

\section{Results and Discussions}

Based on the pathway analyses, the following ten metabolites were identified that matched all of the above-mentioned selection criteria and can be proposed as potential candidates for developing novel antibiotics. Previous identification of the first five metabolites as targets for antibiotic discovery validates our in silico approach and suggests that the five other metabolites could be promising novel candidates as well. The following subsections provide more detailed information on the known or potential role of all ten of these metabolites in therapeutic intervention.

A. Lipid II (N-acetylmuramoyl-L-alanyl-D-glutamyl-meso2,6-diaminoheptane-Dalanyl-D-alanine-diphosphoundecaprenyl-N-acetylglucosamine)

Lipid II is a membrane-anchored peptidoglycan precursor that is essential for bacterial cell wall biosynthesis. It is the target for at least four classes of antibiotics, including glycopeptides, lipopeptides, and lantibiotics [15]. Lipid II is a broad-spectrum target, and antibiotics that interact with lipid II cause bacterial cell death in many ways. Examples include equimolar stoichiometric complex formation with lipid II followed by inhibition of cell wall biosynthesis (plectasin), and permeabilization of membranes by binding to lipid II, followed by assembly and pore formation (nisin). Lipid II is a chemically complex molecule, with a hydrophilic head group that consists of a GlcNAc-MurNAc-pentapeptide linked via a pyrophosphate to a long lipidic bactoprenol tail [16]. Building synthetic analogs of lipid II for antimicrobial testing would require considerable effort [17] Nonetheless, such analogs present an untapped opportunity in the field of antibiotic discovery.

\section{B. meso-diaminopimelate (meso-DAP)}

Meso-DAP is a unique metabolite that functions as a precursor of lysine and as a structural component of the pentapeptide linker in the peptidoglycan layer of most bacterial cell walls, except gram-positive cocci. The metabolite is present in most algae, fungi, and higher plants, but absent in mammals. DAP cross-links provide stability to the cell wall and confer resistance to intracellular osmotic pressure. The four variants of the biosynthetic pathways of meso-DAP differ in the routes leading from tetrahydrodipicolinate to meso-diaminopimelate. The presence of multiple biosynthetic pathways for DAP, at least in some bacteria, is probably an indication of the importance of DAP to bacterial survival. Various DAP analogs are potent antibacterials. For ex- 
ample, 3-chloro-DAP, an inhibitor of DapF, the DAP epimerase that catalyzes the formation of meso-DAP, is active against E. coli. Other inhibitors include 3-methyl-DAP, which may inhibit DAP transport [18]. Phosphono-DAP analogs, which inhibit DAP biosynthesis, are active against a wide range of bacteria. $\gamma$-Methylene-DAP inhibits the growth of E. coli and P. aeruginosa [19].

\section{Pantothenate}

Pantothenic acid (Vitamin B5) is the universal precursor for the synthesis of the 4'-phosphopantetheine moiety of coenzyme A (CoA) and acyl carrier protein (ACP). CoA and ACP play important roles as acyl-group carriers in fatty acid metabolism, the tricarboxylic acid cycle, biosynthesis of polyketides, and several other reactions associated with intermediary metabolism. The de novo biosynthetic pathway to pantothenate consists of four enzymes encoded by panB, panE, panD, and panC; all four are absent in mammals. Bacteria, plants, and fungi synthesize pantothenate de novo from amino acid intermediates, but human beings need to acquire it through diet [20]. Various pantothenate analogs show antibiotic activity against many bacteria, including E. coli and S. aureus [21]. Pantothenate cannot be a broad-spectrum target because many microorganisms that synthesize pantothenate are also capable of absorbing preformed pantothenate from the extracellular environment. For example, pantothenate uptake is mediated by pantothenate permease PanF in E. coli K-12 [22] and pantothenate transporter PanT in S. pneumoniae [23]. Pantothenate, however, can be a promising target for Mycobacterium tuberculosis. Vaccination with a $\triangle$ panCD strain of Mycobacterium tuberculosis elicited an immune response that protected immuno-compromised mice against virulent $M$. tuberculosis more effectively than a BCG vaccination did, without causing widespread infection by itself. A sulfamoyl adenylate inhibitor of $\mathrm{PanC}$ is also being tested for activity in a cell-based assay against Mycobacterium tuberculosis [24].

\section{Biotin}

Biotin or Vitamin $\mathrm{H}$ is a co-factor for a small number of enzymes that facilitate the transfer of $\mathrm{CO}_{2}$ during carboxylation, decarboxylation, and transcarboxylation reactions involved in fatty acid and carbohydrate metabolism. The biotin-requiring enzymes identified to date (including acetyl-CoA carboxylase, 3methylcrotonyl-CoA carboxylase, propionyl-CoA carboxylase, and pyruvate carboxylase) play essential roles in cell metabolism [25]. Biotin is present in microorganisms and higher plants, and absent from mammals, which must acquire it from their diets. Like pantothenate, biotin cannot be considered a broad-spectrum target since a biotin transporter has been found in E. coli, S. aureus, and S. pneumoniae [26]. It can be a potential target for M. tuberculosis, however, as biotin functions as a precursor for synthesis of mycolate, the major cell wall component essential for survival and pathogenesis of $M$. tuberculosis [27] and for degradation of cholesterol, which is an alternative carbon source during their persistence within the human host [28]. Two antibiotics, actithiazic acid and amiclenomycin, irreversibly inactivate $B i o A$, which is involved in biotin biosynthesis and needed for the longterm survival of mycobacteria. Mycobacteria might be able to reverse the effect of such antibiotics by taking up external biotin, although a transporter that could perform this function has not been identified in the annotated genes of $M$. tuberculosis [25]. Biotin antagonists such as 4-(Imidazolidone-2) caproic acid, homobiotin, norbiotin, and hexahydro-2-oxo-4-hydroxybutyl-1-furo- $(3,4)$ imidazole have shown inhibitory effects on $M$. tuberculosis.. a-Dehydrobiotin, a naturally occurring biotin analog, exhibits antimicrobial properties against E. coli, B. subtilis, and several strains of mycobacteria. It is a product of biotin catabolism that coordinately represses the 7,8-diaminopelargonic acid aminotransferase and the dethiobiotin synthetase enzymes [29].

\section{E. Shikimate}

Shikimate leads in three enzymatic reaction steps to the production of chorismate, a key precursor of biosynthesis of several essential metabolites, including aromatic amino acids, ubiquinones, folates, naphthoquinones, menaquinones, and mycobactins. Shikimate is present in algae, higher plants, bacteria, and fungi, but absent in mammals [30]. It could be a broad-spectrum target. Genes for seven enzymes of the shikimate pathway-aroD, aroB, aroK, aroF, aroG, aroE, and $\operatorname{aro} A$-have been shown to be essential for mycobacterial viability. Even in the presence of exogenous supplementation, none of these aro mutants could be obtained in M. tuberculosis [30]. A shikimate analog, (6S)-6-fluoroshikimic acid, acts as a broad-spectrum antibacterial agent. It is active against $E$. coli, presumably because inhibition of aromatic biosynthesis results from the irreversible inhibition of 4-amino-4- deoxychorismate synthase caused by 2 -fluorochorismate [31].

\section{F. L-aspartyl-4-phosphate}

L-aspartyl-4-phosphate is present in plants and bacteria, but absent from mammals. It is produced from ATP-dependent phosphorylation of L-aspartate through a reaction catalyzed by aspartokinase, an enzyme that feeds a branched network of many biochemical pathways, including the biosynthesis of the aspartate family of amino acids: methionine, threonine, lysine, and isoleucine. All these are essential amino acids for humans and are absorbed through diet [32] Consequently, L-aspartyl-4-phosphate is expected to be a broadspectrum target. Transposon site hybridization analysis identified the aspartokinase-encoding (ask) gene as one of the genes required for the growth of M. tuberculosis [33]. In E. coli, unsaturated and fluorinated analogues of aspartyl-4-phosphate act as potential inhibitors of the enzyme aspartate semialdehyde dehydrogenase, which catalyzes the formation of L-aspartate semialdehyde from aspartyl-4-phosphate [34].

\section{G. dTDP- $\alpha$-L-rhamnose}

An L-rhamnosyl residue (a 6-deoxyhexose sugar) plays an important structural role in the cell wall of many human pathogens, including M. tuberculosis and S. typhimurium [35]. A nucleotide linked conjugate of this sugar consisting of deoxythymidine diphosphate dTDP-L-rhamnose is a key intermediate in cell wall synthesis because it donates L-rhamnose. Neither rhamnose nor the genes for its synthesis have been identified in humans. The rhamnose pathway is ubiquitous and highly conserved in both gram-positive and gramnegative bacteria. L-rhamnose is a common component of the O-antigen of lipopolysaccharides (LPS) of gram-negative pathogens such as S. enterica, S. flexneri, and E. coli. L-rhamnose has been found to occupy important anchoring positions in M. tuberculosis, where it covalently links the arabinogalactan to the peptidoglycan layer. Inhibitors (e.g., 2,3,5 trisubstituted-4-thiazolidinone compounds against $\mathrm{RmlC}$ ) of L-rhamnose-synthesizing enzymes have been shown to be active against whole $M$. tuberculosis cells, and the pathway was shown to be essential [36]. L-rhamnose can be used as the sole carbon and energy source by many groups of microorganisms. It cannot be classified as a broad-spectrum target, however, because the utilization of L-rhamnose requires an L-rhamnose-H+ symporter (RhaT) to cross the cytoplasmic membrane in E. coli and S. typhimurium [37]. Since rhamnose has no role in mammalian metabolism, L-rhamnose mimics have been developed as selective antibacterials to inhibit incorporation 


\section{of dTDP-rhamnose into the cell wall [38].}

\section{H. UDP-D-galacto-1, 4-furanose (Galf)}

Galf has been reported in a number of microorganisms, such as bacteria, protozoa, and fungi. It is not present in mammals, and Galf-containing epitopes have been shown to be highly antigenic. Galf residues are formed in nature by a ring contraction of uridine diphosphate galactopyranose (UDP-Galp) to UDP-galactofuranose (UDP-Galf) catalyzed by the enzyme UDP-galactopyranose mutase (UGM). UGM is present in several microorganisms, including E. coli, Mycobacteria, and Klebsiella sp. Galf is transferred from UDP-Galf to the respective glycoconjugate molecules by specific galactofuranosyl transferases. Galf has been shown to be present in numerous structures considered to be essential for virulence in many pathogenic organisms. These include the LPS Oantigen of an increasing number of gramnegative bacteria, the T1-antigen polysaccharide of Salmonella sp., and extracellular or capsular polysaccharides of a variety of both grampositive and gram-negative bacteria. Galf is a critical and abundant component of the arabinogalactan of mycobacteria. Mycobacterial arabinogalactan, a polysaccharide consisting largely of Galf, covalently links the highly impermeable mycolic acid outer layer of the mycobacterial cell wall with the inner layer of peptidoglycan. The metabolism of arabinan, which is directly linked to the Galf component of arabinogalactan, is the target of the proven antituberculosis drug ethambutol [39]. Therefore, Galf metabolism is a particularly promising target in the search for new antimycobacterial drugs. From a focused library of synthetic aminothiazoles, several compounds that block the UGM from K. pneumoniae or M. tuberculosis were identified. A pyrazole compound with a similar structure has been shown to be an inhibitor of UGM from M. tuberculosis and K. pneumonia; it was also effective against $M$. bovis BCG and M. tuberculosis, but it was ineffective against other bacterial strains tested. This compound showed potency against mycobacteria in infected macrophages, but it also exhibited moderate cellular toxicity and was ineffective against nonreplicating persistent mycobacteria [40,41].

\section{I. des-N-acetyl mycothiol/Cys-GlcN-Ins}

The major low-molecular-mass thiol found in the actinomyceteae group of bacteria is mycothiol (MSH; AcCys-GlcNIns). This thiol, which is unique to these organisms, has the structure (1-D-myoinosityl2(N-acetyl-L-cysteinyl) amino-2-deoxy- $\alpha$-D-glucopyranoside. MSH is the functional equivalent of glutathione and is present at millimolar levels in M. tuberculosis. It functions as a reserve of cysteine and is used for the detoxification of alkylating agents, reactive oxygen, nitrogen species, and antibiotics. Mycothiol also acts as a thiol buffer, maintaining the highly reducing environment within the cell and protecting against disulfide stress. MSH biosynthesis is absent in human beings. Current studies indicate that dormant M. tuberculosis cells are metabolically active and therefore must maintain a reducing intracellular redox environment. Since MSH and its disulfide reductase form the thiol redox buffer in mycobacteria, MSH biosynthesis drug targets may be particularly relevant to the treatment of dormant tuberculosis infection [42]. Des- N-acetyl mycothiol or Cys-GlcN-Ins is the immediate precursor of MSH. The enzymes of the MSH biosynthesis are $M \operatorname{sh} A / M \operatorname{sh} A 2, M \operatorname{sh} B, M s h C$, and $M s h D . M \operatorname{shC}$ has been found to be an essential enzyme for producing Cys-GlcN-Ins, which in turn can undergo transacetylation to make N-formyl-Cys-GlcN-Ins (a weak surrogate of MSH) even in the presence of low MSH (in the case of the mshD mutant) [43]. Therefore des- $\mathrm{N}$-acetyl mycothiol may be a better target than MSH. The $m s h A$ deletion mutants are defective in $\mathrm{MSH}$ biosynthesis and ethionamide resistant, as well as slightly defective for growth in immunocompetent mice [44]. The $m s h B$ mutant had a heightened sensitivity to the toxic oxidant cumene hydroperoxide and to rifampin. Viable mutants with the native $m s h C$ gene inactivated could be obtained only when a second copy of $m s h C$ was present, thus indicating that MSH is essential for growth. The $m s h D$ mutant produces only $1 \%$ of normal MSH levels; it produces $\mathrm{N}$-formyl-CysGlcNIns (as a weak surrogate of MSH), but not in sufficient quantities to support normal growth of $M$. tuberculosis under stress conditions such as those found within the macrophage [45].

Dequalinium chloride, an ATP-competitive inhibitor of $M s h C$, has been shown to inhibit the growth of $M$. tuberculosis under aerobic and anaerobic conditions [46]. In vivo, the 1-L-Ins-1-P required for MSH biosynthesis is usually obtained from glucose-6-phosphate by 1-L-inositol-1-phosphate synthase (Ino1). Antisense inhibition of Ino1 in M. tuberculosis results in a marked depletion of intracellular MSH levels and increased sensitivity to vancomycin, rifampicin, and isoniazid. A thioglycosidic analogue of mycothiol has recently been shown to have good specific activity against $M$. tuberculosis [47].

\section{J. Siroheme}

Sulfur and nitrogen metabolism are ancient, essential biosynthetic pathways. Human beings cannot process sulfur and nitrogen directly, but rather depend on bacteria and plants that possess the necessary metabolic pathways to reduce inorganic sulfur and nitrogen to the correct redox state for human consumption. Siroheme is an ironcontaining isobacteriochlorin, a modified tetrapyrrole similar in structure to both heme and chlorophyll, which was discovered in 1973 [48]. It is an unusual but useful prosthetic group of several enzymes, including sulfite and nitrite reductases, which catalyze the six-electron reductions of sulfite to sulfide and nitrite to ammonia, respectively. Assimilatory sulfite reductases are found in bacteria, fungi, and plants, but not in animals, while dissimilatory sulfite reductases are found in diverse sulfate-reducing eubacteria and some species of thermophilic archaebacteria [49]. Siroheme can therefore be considered a broadspectrum target. Siroheme is covalently coupled to an iron-sulfur cluster $([\mathrm{FeS}])$ to form an electronically integrated metallo-co-factor for delivering electrons to a substrate. It is formed by methylation, oxidation, and iron insertion into the tetrapyrrole uroporphyrinogen III (Uro-III). The enzymes catalyzing this pathway have many variations. In some bacteria the transformation of uroporphyrinogenIII into siroheme is catalyzed by three separate enzymes (uroporphyrin III methyltransferase, dihydrosirohydrochlorin dehydrogenase, and sirohydrochlorin ferrochelatase) [50]. In other organisms, such as E. coli and S. enterica, a single trifunctional enzyme (uroporphyrin III C-methyltransferase [multifunctional], CysG) catalyzes all three reactions [51]. In either case, cys $G$ mutants cannot reduce sulfite to sulfide and require a source of sulfide or cysteine for growth. In addition, $C y s G$-mediated methylation of Uro-III is required for de novo synthesis of cobalamin (coenzyme B12) in S. enterica [52]. cysF, encoding an alternative siroheme synthase homologous to $C y s G$, has been identified in K. aerogenes. In contrast, Klebsiella cys $G$ mutants fail to synthesize coenzyme B12. The $c y s F$ gene is absent from the E. coli and S. enterica genomes [53].

\section{Conclusion}

In this study, we have performed in-depth literature mining to find the essential metabolites for a wide range of pathogenic bacteria. The research focused on metabolites for which direct experimental evidence supports the conclusion that these are essential for the growth and survival of the relevant bacteria. To identify potential drug targets, 
Citation: Sarker M, Chopra S, Mortelmans K, Kodukula K, Talcott C, et al. (2011) In Silico Pathway Analysis Predicts Metabolites that are Potential Antimicrobial Targets. J Comput Sci Syst Biol 4: 021-026. doi:10.4172/jcsb.1000071

we have further screened these metabolites to eliminate any found in the human host. Comparative whole genome metabolic pathway analysis was performed computationally using BioCyc Pathway Tools. The results of our analysis were validated when we found literaturebased evidence of antibacterial activity in analogs of lipid II, mesoDAP, pantothenate, biotin, and shikimate. This validation suggests that the remaining five relatively underexplored metabolites from our list are attractive targets for antibiotic discovery. These findings indicate that computational pharmacophore approaches can be useful for in silico antimicrobial design efforts to identify essential metabolite mimics that are similar to known drugs. Our analysis suggests that novel synthetic analogs of the metabolites reported here could provide effective antibiotics. Moreover, novel intervention strategies such as in vitro selection can be used to identify metabolite "scavenging" peptide aptamers as a new class of antibiotics.

\section{Acknowledgments}

The establishment of Center for Advanced Drug Research (CADRE) was made possible by funding support to SRI International from the Commonwealth of Virginia. This work was supported by grant 53123 from the Bill \& Melinda Gates Foundation. Encouragement and guidance from Dr. Walter Moos, Vice President of the Biosciences Division of SRI International, are gratefully acknowledged.

\section{References}

1. Nikaido H (2009) Multidrug resistance in bacteria. Annu Rev Bioche 78:119146

2. Lambert PA (2005) Bacterial resistance to antibiotics: modified target sites. Adv Drug Deliv Rev 57: 1471-1485.

3. ShiveW (1952) Biological activities of metabolite analogues. Annu Rev Microbiol 6: 437-466.

4. Cherkasov A (2006) Can 'Bacterial-Metabolite-Likeness' model improve odds of 'in silico' antibiotic discovery? J Chem Inf Model 46:1214-1222.

5. Masters PA, O'Bryan TA, Zurlo J, Miller DQ, Joshi N (2003) Trimethoprim sulfamethoxazole revisited. Arch Intern Med 163: 402-410.

6. Vilcheze C, Jacobs WR Jr (2007) The mechanism of isoniazid killing: clarity through the scope of genetics. Annu Rev Microbiol 61: 35-50.

7. Rengarajan J, Sassetti CM, Naroditskaya V, Sloutsky A, Bloom BR, et al. (2004) The folate pathway is a target for resistance to the drug para-aminosalicylic acid (PAS) in mycobacteria. Mol Microbiol 53: 275-282.

8. Halouska S, Chacon O, Fenton RJ, Zinniel DK, Barletta RG, et al. (2007) Use of NMR metabolomics to analyze the targets of D-cycloserine in mycobacteria: role of D-alanine racemase. J Proteome Res 6: 4608-4614.

9. Lee ER, Blount KF, Breaker RR (2009) Roseoflavin is a natural antibacterial compound that binds to FMN riboswitches and regulates gene expression. RNA Biol 6: 187-194.

10. Karp PD, Paley S, Romero P (2002) The Pathway Tools software. Bioinformatics 18: $\mathrm{S} 225-232$

11. Paley SM, Karp PD (2006) The Pathway Tools cellular overview diagram and Omics Viewer. Nucleic Acids Res 34: 3771-3778.

12. Karp PD, Ouzounis CA, Moore-Kochlacs C, Goldovsky L, Kaipa P, et al. (2005) Expansion of the BioCyc collection of pathway/genome databases to 160 genomes. Nucleic Acids Res 33: 6083-6089.

13. Romero P, Wagg J, Green ML, Kaiser D, Krummenacker M, et al. (2005) Computational prediction of human metabolic pathways from the complete human genome. Genome Biol 6: R2.

14. Caspi R, Altman T, Dale JM, Dreher K, Fulcher CA, et al. (2010) The MetaCyc database of metabolic pathways and enzymes and the BioCyc collection of pathway/genome databases. Nucleic Acids Res 38: D473-479.

15. Schneider T, Sahl HG (2010) Lipid II and other bactoprenol-bound cell wall precursors as drug targets. Curr Opin Investig Drugs 11: 157-164.
16. Breukink E, de Kruijff B (2006) Lipid II as a target for antibiotics. Nat Rev Drug Discov 5: 321-332.

17. Van Heijenoort $J$ (2007) Lipid intermediates in the biosynthesis of bacterial peptidoglycan. Microbiol Mol Biol Rev 71: 620-635.

18. Baumann RJ, Bohme EH, Wiseman JS,Vaal M, Nichols JS (1988) Inhibition of Escherichia coli growth and diaminopimelic acid epimerase by 3-chlorodiaminopimelic acid. Antimicrob Agents Chemother 32: 1119-1123.

19. Cox RJ (1996) The DAP pathway to lysine as a target for antimicrobial agents Nat Prod Rep 13: 29-43.

20. Sambandamurthy VK, Wang X, Chen B, Russell RG, Derrick S, et al. (2002) A pantothenate auxotroph of Mycobacterium tuberculosis is highly attenuated and protects mice against tuberculosis. Nat Med 8: 1171-1174.

21. Mcllwain H (1942) Bacterial inhibition by metabolite analogues: Analogues of pantothenic acid. Biochem J 36: 417-427.

22. Jackowski S, Alix JH (1990) Cloning, sequence, and expression of the pantothenate permease (panF) gene of Escherichia coli. J Bacteriol 172: 3842 3848 .

23. Rodionov DA, Hebbeln P, Eudes A, ter Beek J, Rodionova IA, et al. (2009) A novel class of modular transporters for vitamins in prokaryotes. $\mathrm{J}$ Bacterio 191: $42-51$.

24. Ciulli A, Scott DE, Ando M, Reyes F, Saldanha SA, et al. (2008) Inhibition of Mycobacterium tuberculosis pantothenate synthetase by analogues of the reaction intermediate. Chembiochem 9: 2606-2611.

25. Mann S, Ploux O (2006) 7,8-Diaminoperlargonic acid aminotransferase from Mycobacterium tuberculosis, a potential therapeutic target. Characterization and inhibition studies. FEBS J 273: 4778-4789.

26. Hebbeln P, Rodionov DA, Alfandega A, Eitinger T (2007) Biotin uptake in prokaryotes by solute transporters with an optional ATPbinding cassettecontaining module. Proc Natl Acad Sci U S A 104: 2909-2914.

27. Kurth DG, Gago GM, de la Iglesia A, Bazet Lyonnet B, Lin TW, et al. (2009) ACCase 6 is the essential acetyl-CoA carboxylase involved in fatty acid and mycolic acid biosynthesis in mycobacteria. Microbiology 155: 2664-2675.

28. Savvi S, Warner DF, Kana BD, McKinney JD, Mizrahi V, et al. (2008) Functiona characterization of a vitamin B12-dependent methylmalonyl pathway in Mycobacterium tuberculosis: implications for propionate metabolism during growth on fatty acids. J Bacteriol 190: 3886-3895.

29. Piffeteau A, Dufour MN, Zamboni M, Gaudry M, Marquet A (1980) Mechanism of the antibiotic action of alpha-dehydrobiotin. Biochemistry 19: 3069-3073.

30. Dosselaere F, Vanderleyden J (2001) A metabolic node in action: chorismateutilizing enzymes in microorganisms. Crit Rev Microbiol 27: 75-131.

31. Bulloch EM, Jones MA, Parker EJ, Osborne AP, Stephens E, et al. (2004) Identification of 4-amino-4-deoxychorismate synthase as the molecular target for the antimicrobial action of (6s)-6-fluoroshikimate. J Am Chem Soc 126 9912-9913.

32. Viola RE (2001) The central enzymes of the aspartate family of amino acid biosynthesis. Acc Chem Res 34: 339-349.

33. Sassetti CM, Boyd DH, Rubin EJ (2003) Genes required for mycobacteria growth defined by high density mutagenesis. Mol Microbiol 48: 77-84.

34. Cox RJ, Gibson JS, Hadfield AT (2005) Design, synthesis and analysis of inhibitors of bacterial aspartate semialdehyde dehydrogenase. Chembiochem 6: $2255-2260$.

35. Dong C, Beis K, Giraud MF, Blankenfeldt W, Allard S, et al. (2003) A structural perspective on the enzymes that convert dTDP-d-glucose into dTDPIrhamnose. Biochem Soc Trans 31: 532-536.

36. Babaoglu K, Page MA, Jones VC, McNeil MR, Dong C, et al. (2003) Nove inhibitors of an emerging target in Mycobacterium tuberculosis; substituted thiazolidinones as inhibitors of dTDP-rhamnose synthesis. Bioorg Med Chem Lett 13: 3227-3230. 
Citation: Sarker M, Chopra S, Mortelmans K, Kodukula K, Talcott C, et al. (2011) In Silico Pathway Analysis Predicts Metabolites that are Potential Antimicrobial Targets. J Comput Sci Syst Biol 4: 021-026. doi:10.4172/jcsb.1000071

37. Tate CG, Muiry JA, Henderson PJ (1992) Mapping, cloning, expression, and sequencing of the rhaT gene, which encodes a novel Lrhamnose- $\mathrm{H}+$ transport protein in Salmonella typhimurium and Escherichia coli. J Biol Chem 267: 6923-6932.

38. Estevez JC, Saunders J, Besra GS, Brennan PJ, Nash RJ, et al. (1996) Mimics of L-Rhamnose: Synthesis of CGlycosides of L-Rhamnofuranose and an alpha-azidoester as divergent intermediates for combinatorial generation of rhamnofuranose libraries. Tetrahedron: Asymmetry 7: 383-386.

39. Pedersen LL, Turco SJ (2003) Galactofuranose metabolism: a potential target for antimicrobial chemotherapy. Cell Mol Life Sci 60: 259-266.

40. Borrelli S, Zandberg WF, Mohan S, Ko M, Martinez-Gutierrez F, et al. (2010) Antimycobacterial activity of UDP-galactopyranose mutase inhibitors. Int $J$ Antimicrob Agents 36: 364-368.

41. Dykhuizen EC, May JF,Tongpenyai A, Kiessling LL (2008) Inhibitors of UDPgalactopyranose mutase thwart mycobacterial growth. J Am Chem Soc 130: 6706-6707.

42. Newton GL, Buchmeier N, Fahey RC (2008) Biosynthesis and functions of mycothiol, the unique protective thiol of Actinobacteria. Microbiol Mol Biol Rev 72: 471-494.

43. Sareen D, Newton GL, Fahey RC, Buchmeier NA (2003) Mycothiol is essentia for growth of Mycobacterium tuberculosis Erdman. J Bacteriol 185: 6736-6740.

44. Vilcheze C, Av-Gay Y, Attarian R, Liu Z, Hazbon MH, et al. (2008) Mycothio biosynthesis is essential for ethionamide susceptibility in Mycobacterium tuberculosis. Mol Microbiol 69: 1316-1329.
45. Buchmeier NA, Newton GL, FaheyRC (2006) A mycothiol synthase mutant of Mycobacterium tuberculosis has an altered thioldisulfide content and limited tolerance to stress. J Bacteriol 188: 6245-6252.

46. Gutierrez-Lugo MT, Baker H, Shiloach J, Boshoff H, Bewley CA (2009) Dequalinium, a new inhibitor of Mycobacterium tuberculosis mycothiol ligase identified by high-throughput screening. J Biomol Screen 14: 643-652.

47. Witczak ZJ, Culhane JM (2005) Thiosugars: new perspectives regarding availability and potential biochemical and medicinal applications. Appl Microbio Biotechnol 69: 237-244.

48. Murphy MJ, Siegel LM (1973) Siroheme and sirohydrochlorin. The basis for a new type of porphyrin-related prosthetic group common to both assimilatory and dissimilatory sulfite reductases. J Biol Chem 248: 6911-6919.

49. Crane BR, Getzoff ED (1996) The relationship between structure and function for the sulfite reductases. Curr Opin Struct Biol 6: 744-756.

50. Johansson P, Hederstedt L (1999) Organization of genes for tetrapyrrole biosynthesis in gram--positive bacteria. Microbiology 145: 529-538.

51. Warren MJ, Bolt EL, Roessner CA, Scott AI, Spencer JB et al. (1994) Gene dissection demonstrates that the Escherichia coli cysG gene encodes a multifunctional protein. Biochem J 302: 837-844.

52. Woodcock SC, Raux E, Levillayer F, Thermes C, Rambach A, et al. (1998) Effect of mutations in the transmethylase and dehydrogenase/chelatase domains of sirohaem synthase (CysG) on sirohaem and cobalamin biosynthesis. Biochem J 330: 121-129.

53. Kolko MM, Kapetanovich LA, LawrenceJG (2001) Alternative pathways for siroheme synthesis in Klebsiella aerogenes. J Bacteriol 183: 328-335. 